\title{
Stable Laws and the Number of Ordinary
}

\author{
P.M. Mazurkin ${ }^{*}$ \\ Doctor of Engineering Science, Academician of RANS, member of EANS, Volga Region State Technological University, Russia \\ *Corresponding author: kaf_po@mail.ru
}

Received February 08, 2014; Revised February 17, 2014; Accepted February 20, 2014

\begin{abstract}
Power total number of primes from the discharge of the decimal system is identified by the law of exponential growth with 14 fundamental physical constants. Model obtained on the parameters of the physical constants, proved less of the error and it gives more accurate predictions of the relative power of the set of prime numbers. The maximum absolute error of power (the number of primes), the traditional number is three times higher than suggested by us complete a number of prime numbers. Therefore, the traditional number $2,3,5,7, \ldots$ is only a special case. The transformation $\ln 10=2,302585 \ldots$ it was a rough rounded, leading to false identification of physico-mathematical regularities of different series of prime numbers. Model derived from physical constants, proved more accurate than the relative accuracy, and it gives more accurate predictions of the relative power of the set of prime numbers with increasing discharge the decimal number system.
\end{abstract}

Keywords: primes, total number, physical constants, the relationship

Cite This Article: P.M. Mazurkin, "Stable Laws and the Number of Ordinary." Applied Mathematics and Physics, vol. 2, no. 2 (2014): 27-32. doi: 10.12691/amp-2-2-1.

\section{Introduction}

Prime number - is a natural number $\mathrm{N}=\{0,1,2,3,4,5,6, \ldots\}$ that has two positive divisors: one and itself.

There are several variants of distribution or a series of prime numbers (SPN):

1) finite number of critical primes $P=\{0,1,2\}$;

2) non-critical prime numbers $P=\{3,5,7,11,13,17, \ldots\}$;

3) the traditional [1] number of primes $a(n)=\{2,3,5,7,11.13 .17, \ldots\}$ with order (serial number) $n=\{1,2,3, \ldots\}$, which was considered by many scientists and by Riemann;

4) part series of prime numbers $P=\{1,2,3,5,7,11,13,17, \ldots\}$;

5) the total number of prime numbers $\mathrm{P}=\{0,1,2,3,5,7,11,13,17, \ldots\}$ that are equivalent row $\mathrm{N}$.

The literature focuses on $\mathrm{SPN}_{3}$, and we did not find sufficient publications on the analysis of $\mathrm{SPN}_{4}$ and other ranks have been proposed by us. In this reader a series of five articles examined $\mathrm{SPN}_{1}, \mathrm{SPN}_{2} \mathrm{SPN}_{5}$ and compared with evidence $\mathrm{SPN}_{3}$.

\section{Methodology}

The main type of natural numbers are in $(0 ; \infty)$. If we miss this kind of integers due to rejection of negative numbers. For convenience, we will use mathematical analysis, recording the results of calculations in the form of rational numbers just to prove the Riemann hypothesis.
Quantitative analysis is a leap to mind the actual (real) numbers, according to the scheme $\mathrm{P} \subset \mathrm{N} \subset R \not \subset C$ without taking into account the complex numbers, but with irrational numbers such as $e=2,71 \ldots$ and $\pi=3,14 \ldots$ (18 characters after the decimal point in the software environment CurveExpert) and other fundamental constants.

In the analysis of stable laws have been applied [3] to the distribution of prime numbers.

\section{Biotechnical Law and Its Fragments}

Under the scheme "from the simple to the complex structure" in Table 1 are all stable laws are used to construct formulas biotech laws. Generalizing formula is biotech law [3]. Most often, the sum of two biotech laws constitutes a deterministic allocation model.

Formula, together with a finite set SPN runs in a software environment CurveExpert for parameter identification of a stable law and wave patterns. Search for the model parameters is called the structural-parametric identification. For the processes of behavior of living and/or inert substances (according to V.I. Vernadsky) parameters $a, b, c, d$ biotech law and its fragments may approach to the fundamental physical constants, and it has been shown in the distribution of chemical elements [4].

\section{Power Series of Prime Numbers}

According to [1] $\mathrm{SPN}_{3}$ and our calculations on $\mathrm{SPN}_{5}$ in Table 2 shows the cardinal numbers and their relationships $\mathrm{SPN}_{5} / \mathrm{SPN}_{3}$. 
Table 1. Mathematical constructs in the form of stable laws to build a statistical model

\begin{tabular}{|c|c|}
\hline Fragments without previous history of the phenomenon or process & Fragments from the prehistory of the phenomenon or process \\
\hline $\begin{array}{c}y=a x-\text { law of linear growth or decline (with a negative sign in } \\
\text { front of the right side of this formula) }\end{array}$ & $\begin{array}{l}y=a \text { - the law does not impact adopted by the variable on the indicator, which } \\
\text { has a prehistory of up period (interval) measurements }\end{array}$ \\
\hline $\begin{array}{c}y=a x^{b} \text { - exponential growth law (law of exponential } \\
\text { death ) } y=a x^{-b} \text { is not stable because of the appearance of } \\
\text { infinity at zero explanatory variable }\end{array}$ & $\begin{array}{c}y=a \exp ( \pm c x) \text { - Law of Laplace in mathematics (Zipf in biology, Pareto in } \\
\text { economics, Mandelbrot in physics) exponential growth or loss respect to which } \\
\text { the Laplace created a method of operator calculus }\end{array}$ \\
\hline $\begin{array}{c}y=a x^{b} \exp (-c x) \text { - biotech law (law of life skills) in a } \\
\text { simplified form }\end{array}$ & $y=a \exp \left( \pm c x^{d}\right)$ - law of exponential growth or death (P.M. Mazurkin) \\
\hline
\end{tabular}

Note. In bold the full design of stable laws.

Table 2. The relative cardinal number the increase in the capacity (quantity) of prime numbers

\begin{tabular}{|c|c|c|c|c|c|c|c|}
\hline \multirow{2}{*}{$\begin{array}{c}\text { Discharge } \\
i_{10}\end{array}$} & \multirow{2}{*}{$\begin{array}{c}\text { The power of numbers } \\
\mathrm{N}=\{0,1,2,3, \ldots\} \\
x\end{array}$} & \multicolumn{2}{|c|}{ Traditional SPN $_{3}[1]$} & \multicolumn{2}{|c|}{ Full SPN } & \multicolumn{2}{|c|}{$\mathrm{SPN}_{5} / \mathrm{SPN}_{3}, \%$} \\
\hline & & $\begin{array}{c}\text { Power } \\
\pi(x)\end{array}$ & $x / \pi(x)$ & $\begin{array}{c}\text { Power } \\
\pi(x)\end{array}$ & $x / \pi(x)$ & $\pi(x)$ & $x / \pi(x)$ \\
\hline 1 & 10 & 4 & 2.5 & 6 & 1.6667 & 150,00 & 66,67 \\
\hline 2 & 100 & 25 & 4.0 & 27 & 3.7037 & 108,00 & 92,59 \\
\hline 3 & 1000 & 168 & 6.0 & 170 & 5.8824 & 101,19 & 98.04 \\
\hline 4 & 10000 & 1229 & 8.1 & 1231 & 8.1235 & 100,16 & 100,29 \\
\hline 5 & 100000 & 9592 & 10.4 & 9594 & 10.4232 & 100,02 & 100,22 \\
\hline 6 & 1000000 & 78498 & 12.7 & 78500 & 12.7389 & 100,00 & 100,31 \\
\hline 7 & 10000000 & 664579 & 15.0 & 664581 & 15.0471 & 100,00 & 100,31 \\
\hline 8 & 100000000 & 5761455 & 17.4 & 5761457 & 17.3567 & 100,00 & 99,75 \\
\hline 9 & 1000000000 & 50847534 & 19.7 & 50847536 & 19.6666 & 100,00 & 99,83 \\
\hline 10 & 10000000000 & 455052512 & 22.0 & 455052514 & 21.9755 & 100,00 & 99,89 \\
\hline
\end{tabular}

In the first digit decimal numbers the difference between a full and traditional rows of simple number is equal to $150 \%$. The relative cardinal number is the maximum 100,31 at $i_{10}=6$ and minimum 66.67 at $i_{10}=1$. What SPN better? In advance, we say that $\mathrm{SPN}_{5}$.

\section{Traditional SPN}

With the increase in decimal place of natural numbers the increase in the relative cardinal number of the set of prime numbers with a capacity of more than 455 million occurs (Figure 1) by a deterministic model of the law of exponential growth.

$$
x / \pi(x)=0,00066575 \exp \left(8,10285 i_{10}^{0,10893}\right) .
$$

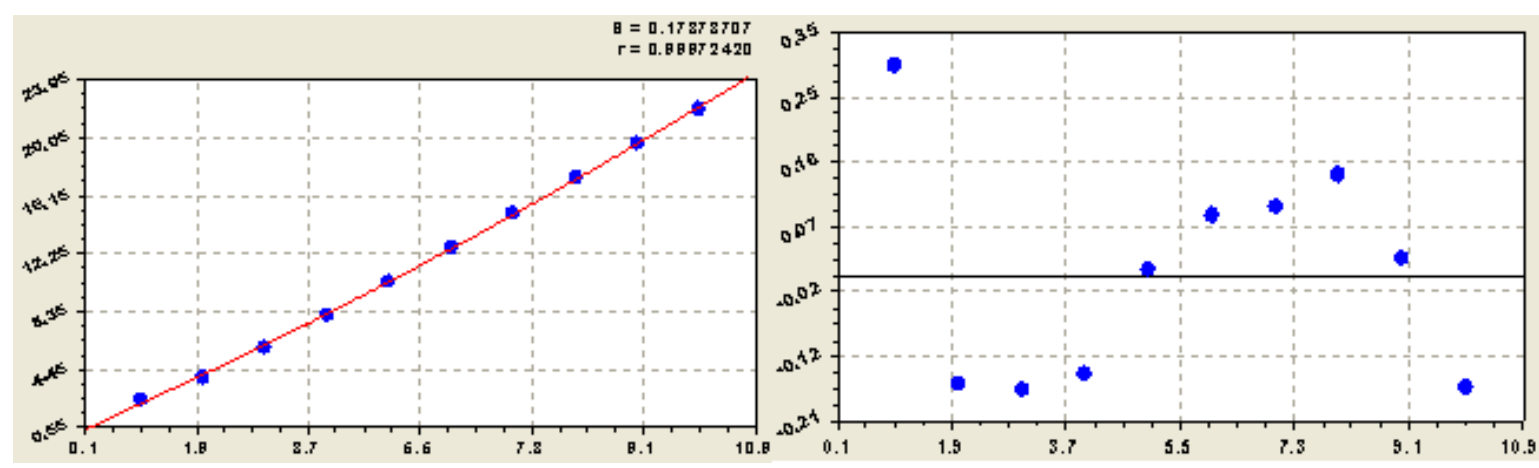

Figure 1. The schedule of the law of exponential growth (1) the relative power and remains after it: $S$ - dispersion; $r$ - correlation coefficient

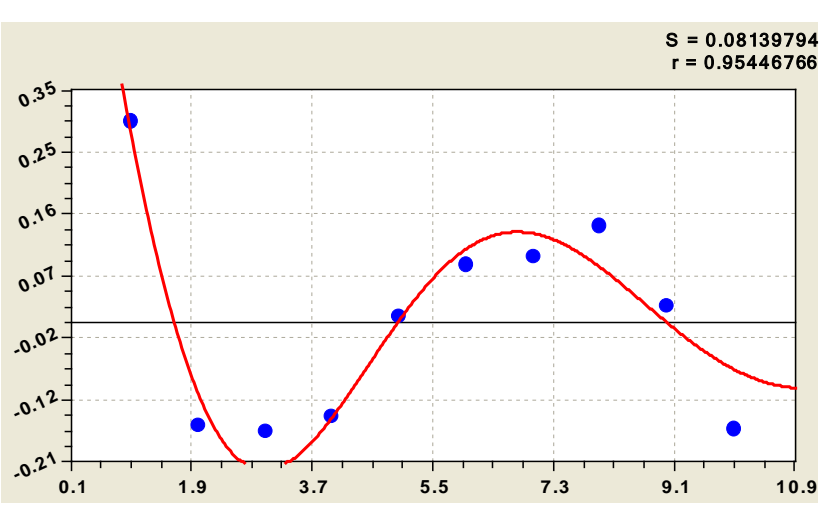

Figure 2. Balance diagram for the model (2)
On the balances was obtained (Figure 2) of the wavelet function (described in the second article)

$$
\begin{aligned}
& \varepsilon=88,26937 \exp \left(-5,36239 i_{10} 0,098706\right. \\
& \cos \left(\pi i_{10} /\left(\begin{array}{l}
0,59537 \\
+1,47125 i_{10} 0,27860
\end{array}\right)-0,67755\right) .
\end{aligned}
$$

The law of exponential death before the cosine function shows half of the amplitude of the oscillatory perturbations of power $\mathrm{SPN}_{3}$. Because of the high value of the remainder for $i_{10}=1$, we have that zero discharge is theoretically possible number of prime numbers must be 88 .

Combining formulas (1) and (2) gives the binomial model with the wave function (Figure 3 ) of the form 


$$
\begin{aligned}
x / \pi(x) & =0,00074272 \exp \left(8,15289 i_{10}^{0,10111}\right) \\
& +956,514 \exp \left(-5,28998 i_{10}^{0,21896}\right) \\
& \left.\cos \left(\pi i_{10} /\left(\begin{array}{l}
-0,14154+ \\
15,52749 i_{10}
\end{array}\right)^{-0,33681}\right)+1,38397\right) .
\end{aligned}
$$

Top of the wave has moved up to 957 prime numbers with zero discharge of the decimal system. In addition, under the function of the cosine of half-cycle fluctuations has changed: the beginning shifted to the first digit of the negative numbers. Half-life increases sharply, and the intensity parameter of death -0.33681 shows anomalous behavior of the model (3).

From the graph in Figure 3 we can see that the shape of the curve repeats part of the curve of the Riemann zetafunction, located in the positive area of complex numbers.

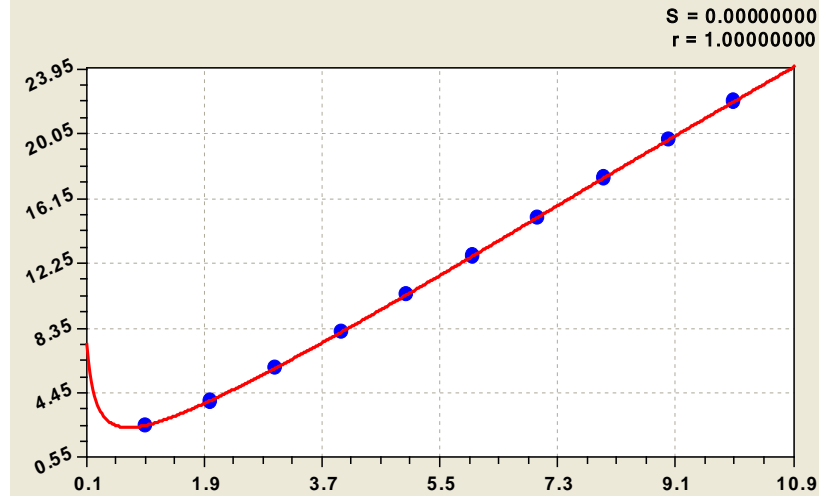

Figure 3. The relative power of the traditional series

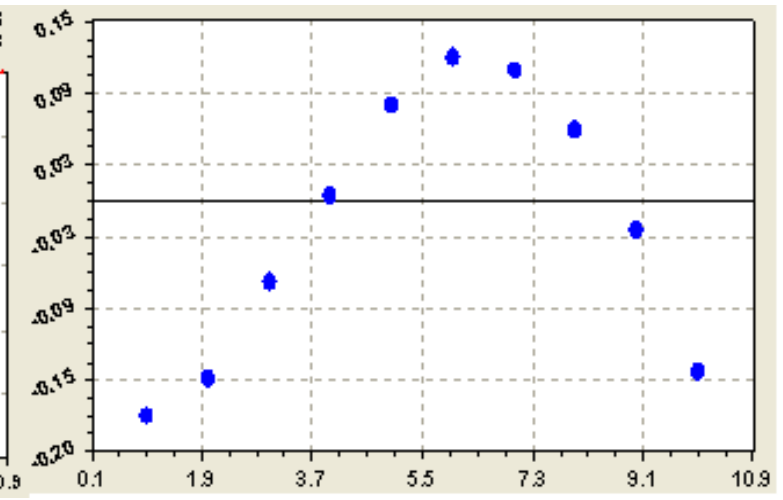

Figure 4. Schedule of the law of exponential growth (4) and residues from him

\section{Full Range}

This $\mathrm{SPN}_{5}$ received a deterministic pattern (Figure 4) the type of

$$
x / \pi(x)=1,50030 \cdot 10^{-24} \exp \left(55,46724 i_{10}{ }^{0,019036}\right) .
$$

Residues have a relatively smooth swing and determined (Figure 5) by the formula:

$$
\begin{aligned}
\varepsilon= & -0,20751 \exp \left(-0,16759 i_{10}^{0,48624}\right) \\
& \cos \left(\pi i_{10} /\left(\begin{array}{l}
8,19322 \\
-0,31718 i_{10} 0,99304
\end{array}\right)-0,22080\right) .
\end{aligned}
$$

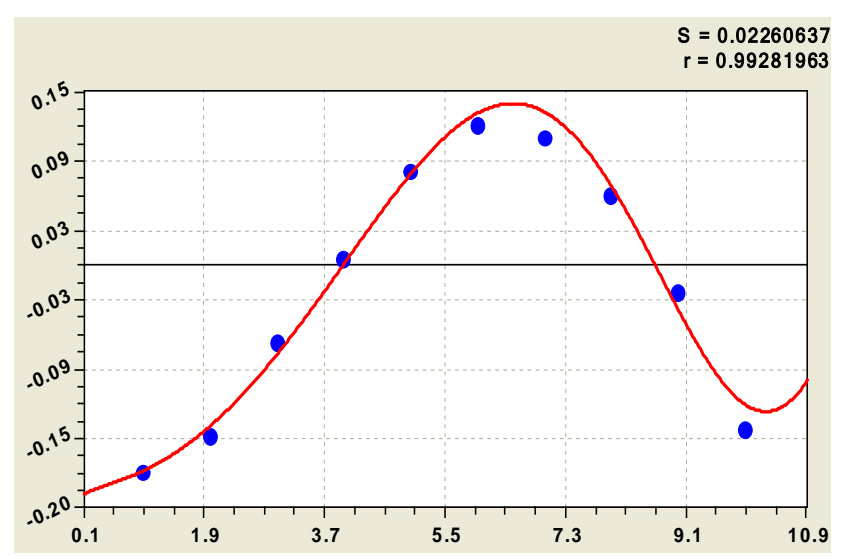

Figure 5. График остатков по модели (5)

In a number of natural numbers $0,1,2,3,4,5,6,7,8,9$, there are six prime numbers, three of which $(0,1,2)$ critical (negative sign before the formula vibration), and three numbers $(3,5,7)$ - a non-critical. At zero there is no prime numbers, so the law of exponential growth begins with a small actual (real) numbers $1,50030 \cdot 10^{-24}$, and 0.20751 .

In formula (5), half of the amplitude of the perturbations of the power $\mathrm{SPN}_{5}$ has the numerical value of all 0,20751. The initial half-life 8,19322 damped oscillations approaching 8.

We express the frequency response $8,19322-0,31718 i_{10}{ }^{0,99304}$ of the oscillatory disturbance is half-wave decline, i.e. with increasing discharge $i_{10}$ is the growth rate fluctuations on the scale of natural numbers $\mathrm{N}=\{0,1,2,3, \ldots\}$, and it - the effect of the tremor.

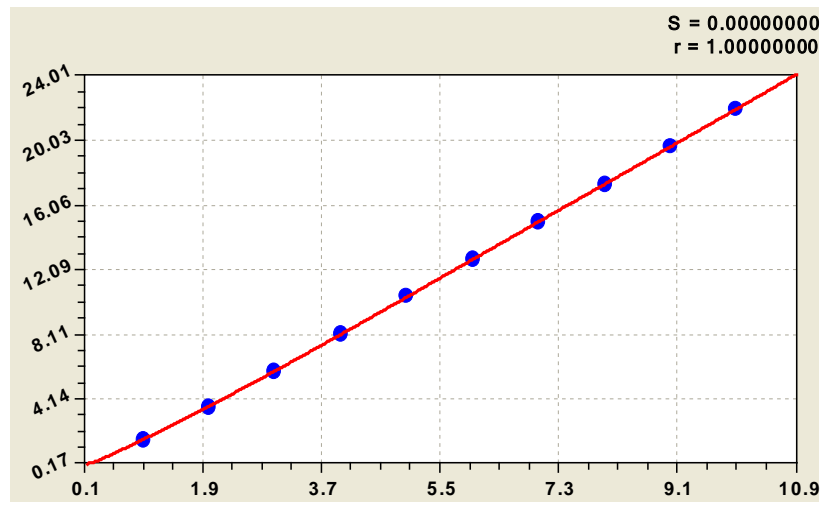

Figure 6. The relative power of the complete series

The general equation (Figure 6) is characterized by binomial formula 


$$
\begin{aligned}
x / \pi(x)= & 1,49766 \cdot 10^{-24} \exp \left(55,46556 i_{10} 0,019025\right) \\
& -0,18905 \exp \left(-0,0032736 i_{10} 1,00713\right) \\
& \cos \left(\pi i_{10} /\left(\begin{array}{l}
7,40869 \\
-0,23358 i_{10} 0,61848
\end{array}\right)-0,028862\right) .
\end{aligned}
$$

Remains of the formula (6) are so small that, as seen in the upper right corner in Figure 6, the variance of the residual is zero and the correlation coefficient is unity.

Comparison of the remains of the formulas (3) and (6) shown in Figure 7.
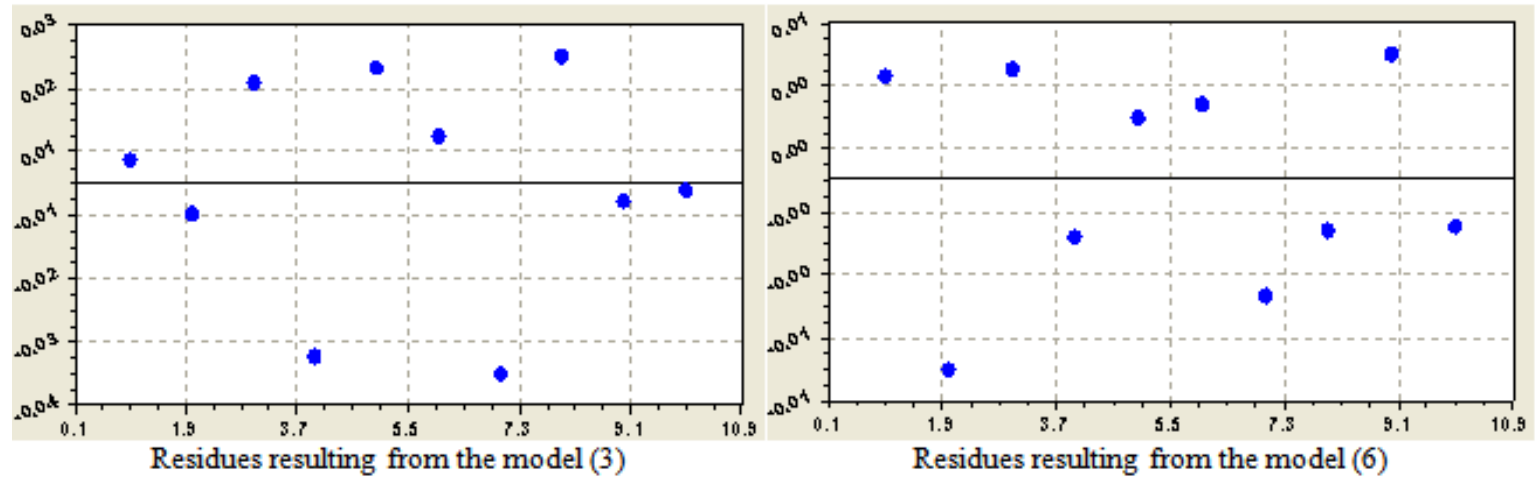

Figure 7. Graphs of the absolute error the laws of growth of capacity of prime numbers

The maximum absolute error of power (the number of primes), the traditional number is three times higher compared to the total number of primes.

Then it turns out that the traditional number is only a special case.

\section{Do not Change the Scale of Reference of Natural Primes}

This recommendation for the future in the study of prime numbers comes from the fact that, from Riemann used the natural logarithm and are looking for an empirical formula [1]. To quote from an article by Don Zagier:

"Apparently (see Table 2.), that the ratio of $\mathrm{x}$ to $\pi(\mathrm{x})$ the transition from a given degree of ten to follow all the time increases to about 2.3. Mathematics is recognizable among the $2.3 \log 10$ (of course, to base e). The result suggested that the $\pi(x) \sim|x / \ln x|$, where the sign $\sim$ means that the ratio of their expressions are connected with $x$ tends to 1 . This asymptotic equation, first proved in 1896, is now the law of distribution of prime numbers. Gauss, the greatest of mathematicians, discovered this law in the age of fifteen, studying tables of primes contained in the gift to him a year before the table of logarithms".

We were not too lazy to check the statement "the ratio of $\mathrm{x}$ to $\pi(\mathrm{x})$ in the transition from the present level of ten to follow all the time increases by about 2.3 " and the results of the calculations resulted in Table 3. Here, the number 2.30 in $\mathrm{SPN}_{3}$ not (if there is, the approximation error to 2.30 at $100(2.5-2.3) / 2.3=8.70 \%$, which is very much), but there is an aspiration to 1 . At the same time the full range of gives at the beginning of the interval of digits in a larger multiplicity 2,22 (error of $3.47 \%$ ).

Equal to the power of two sets $\mathrm{SPN}_{3}$ and $\mathrm{SPN}_{5}$ can be considered, starting with the digits $i_{10} \geq 9$ in decimal notation.

With the growth of $x$ a true statement is the convergence to 1 . For this purpose we identify the law of death (in a general form of Table 1) according to the statistical data of Table 3 .
Table 3. The multiplicity of cardinal number

\begin{tabular}{|c|c|c|c|c|}
\hline \multirow{2}{*}{$\begin{array}{c}\text { Discharge } \\
i_{10}\end{array}$} & \multicolumn{2}{|c|}{ Private $\mathrm{SPN}_{3}[1]$} & \multicolumn{2}{c|}{ Full $\mathrm{SPN}_{5}$} \\
\cline { 2 - 5 } & $x / \pi(x)$ & multiplicity & $x / \pi(x)$ & multiplicity \\
\hline 1 & 2.5 & - & 1.6667 & - \\
\hline 2 & 4.0 & 1.60 & 3.7037 & 2.22 \\
\hline 3 & 6.0 & 1.50 & 5.8824 & 1.59 \\
\hline 4 & 8.1 & 1.35 & 8.1235 & 1.38 \\
\hline 5 & 10.4 & 1.28 & 10.4232 & 1.28 \\
\hline 6 & 12.7 & 1.22 & 12.7389 & 1.22 \\
\hline 7 & 15.0 & 1.18 & 15.0471 & 1.18 \\
\hline 8 & 17.4 & 1.16 & 17.3567 & 1.15 \\
\hline 9 & 19.7 & 1.13 & 19.6666 & 1.13 \\
\hline 10 & 22.0 & 1.12 & 21.9755 & 1.12 \\
\hline
\end{tabular}

For the full range of the obtained formula

$$
\begin{aligned}
& \operatorname{card}\left(x_{i} / \pi\left(x_{i}\right) /\left(x_{i-1} / \pi\left(x_{i-1}\right)\right)\right. \\
= & 1,09980+1788,3968 \exp \left(-6,20754 i_{10}{ }^{0,24956}\right) .
\end{aligned}
$$

Equation (7) shows that the ratio of cardinal numbers will not come near to the unit and can only reach the values of the 1,0998 .

From the article [1] reads: «After more than a careful and complete calculation, Legendre in 1808 found that particularly good approximation is obtained if we subtract from $\ln x$ is not 1 , but 1.08366, i.e. $\pi(x) \sim$ $|x /(\ln x-1,08366)| »$. In the formula (7) the constant 1.09980 is little different.

Thus, number of prime numbers, the power has been studied in a number system with base $e=2.718281828$..... It is known that this system has the greatest density of information recording and refers to the nonintegral positional systems. But non-integers do not belong to the natural numbers $\mathrm{N}$, let alone to a series of prime numbers $a(n)=\{2,3,5,7,11.13 .17, \ldots\}$.

Thus, the transformation $\ln 10=2,302585 \ldots$ it was a rough rounded, leading to false identification of physico- 
mathematical regularities of different series of prime numbers.

With "easy" hands Gauss in mathematics, vigorously developed the theory of approximation, which made it possible to linearize the scale of the abscissa and ordinate in terms of $\ln x$ and $\ln y$. Thus is the fundamental transformation of the statistical data presented at the beginning of the decimal system, in logarithmic. As a result, the closed form of design patterns that are not only difficult to understand, but they have lost and the visibility of graphics and even more so - the physical representation. Therefore, we continue to recommend in its publications to readers an open system of mathematical constructs according to the laws of Table 1.

\section{Fundamental Constants}

Formulas from Table 1 gives the identification of fundamental physical constants to the parameters $a, b, c, d$. Processes themselves are unknown.
Carefully consider the formula (4), and compare the values of parameters of the mathematical model with the fundamental constants. Recall that Don Zagier [1] analyzed (see Table 2) a very large number of natural numbers $\tilde{\mathrm{N}}=\left\{0,1,2,3, \ldots, 10^{10}\right\}$ with a finite number $a(n)=\{2,3,5,7,11.13 .17, \ldots\}$ of prime numbers and gave them a set of up $\pi(x) \rightarrow 455052512$.

We put forward a hypothesis (Table 4): with an increase in the relative power of the total number of prime numbers, the parameters of the model (4) will tend to the fundamental constant [5].

To a first approximation we replace the law (4) to the physical equivalent to the formula

$$
x / \pi(x)=\frac{\sqrt{5}+1}{2 \cdot 10} \frac{\mu_{p}}{\mu_{N}} \mu_{B} e^{\left(m_{e} \sigma_{a} \frac{g_{n}}{10} \frac{m_{p}}{m_{n}}\right) i_{10} \frac{4}{\pi} c_{2}\left(\frac{\mu_{e}}{\mu_{B}} g_{e}-1\right)^{8}},
$$

legend of the model parameters (8) are given in Table 4 (10 - radix).

Table 4. Comparison of parameters of the model (4) of power $\mathrm{SPN}_{5}$ with the fundamental physical constants

\begin{tabular}{|c|c|c|c|c|c|}
\hline \multicolumn{3}{|c|}{ Parameter of the first term of the statistical model (6) } & \multicolumn{2}{|c|}{$\begin{array}{l}\text { The fundamental } \\
\text { physical constant }\end{array}$} & \multirow{2}{*}{$\begin{array}{l}\text { The multiplicity to a } \\
\text { parameter of the model (4) }\end{array}$} \\
\hline Type & Name & Value & Name & Value & \\
\hline \multicolumn{2}{|c|}{ the number of time } & 18 characters* & Number of Napier & $e=2,71828 \ldots$ & $\approx 1$ \\
\hline \multirow{3}{*}{$\begin{array}{l}\text { Trend (tendency) } \\
\text { of prime numbers }\end{array}$} & $\begin{array}{l}\text { Initiation of a series } \\
\text { of prime numbers }\end{array}$ & $1,50030 \cdot 10^{-24}$ & Bohr magneton & $\mu_{B}=9,27402 \cdot 10^{-24}$ & $6,1814 \rightarrow 10 \phi^{-1}$ \\
\hline & $\begin{array}{c}\text { Active growth of } \\
\text { power }\end{array}$ & 55,46724 & $\begin{array}{l}\text { Electron mass } \\
(\mathrm{amu}) \times 10^{-4}\end{array}$ & $m_{e}=5,485799$ & $\begin{array}{c}55,58486=m_{e} \sigma_{=} \\
1,0021105\end{array}$ \\
\hline & $\begin{array}{c}\text { The growth rate of } \\
\text { power }\end{array}$ & 0,019036 & $\begin{array}{c}\text { Radiation: a second } \\
\text { constant }\end{array}$ & $c_{2}=0,0143877$ & $0,75582 \rightarrow \pi / 4$ \\
\hline \multicolumn{2}{|c|}{ The number of harmony } & 18 characters* & $\begin{array}{c}\text { Golden section } \\
\phi=1,61803 \ldots\end{array}$ & $\phi^{-1}=0,61803 \ldots$ & $\approx 1$ \\
\hline \multirow{2}{*}{$\begin{array}{l}\text { Parameters of the } \\
\text { Earth }\end{array}$} & Atmosphere & exactly & Standard atmosphere & $\sigma_{a}=101325$ & 1 \\
\hline & Gravitation & \multicolumn{2}{|c|}{ The acceleration of gravity (standard) } & $g_{n}=9,80665$ & 1 \\
\hline \multirow{6}{*}{ Atom } & \multirow{2}{*}{ Proton } & \multicolumn{2}{|c|}{ Magnetic moment / nuclear magneton } & $\mu_{p} / \mu_{N}=2,7928474$ & $\approx 1$ \\
\hline & & \multicolumn{2}{|c|}{ Mass of the proton (amu) } & $m_{p}=1,00727647$ & $\approx 1$ \\
\hline & Noutmon & \multicolumn{2}{|c|}{ Magnetic moment of the neutron } & $\mu_{n}=0,96623707$ & $\approx 1$ \\
\hline & ग्रeutron & \multicolumn{2}{|c|}{ Mass of the neutron (near.) } & $m_{n}=1,0086649$ & $\approx 1$ \\
\hline & Floot & \multicolumn{2}{|c|}{ Magnetic moment of / Bohr magneton } & $\mu_{e} / \mu_{B}=1,00115965$ & $\approx 1$ \\
\hline & & \multicolumn{2}{|c|}{ Anomaly magnetic moment } & $g_{e}=2,0023193$ & $\approx 1$ \\
\hline \multicolumn{2}{|c|}{ Number of space } & 18 characters* & $\begin{array}{l}\text { Number Of Archimedes } \\
\qquad / 4 \approx 0,78540\end{array}$ & $\pi=3,14159 \ldots$ & $\approx 1$ \\
\hline
\end{tabular}

Note. $*$ In the mathematical environment CurveExpert the possibility of representing irrational numbers.

\section{The Law with the Fundamental Constants}

After substituting the fundamental physical constants in Table 4 we write the model (8) as a law of exponential growth

$$
x / \pi(x)_{f}=4,1908462 \cdot 10^{-24} \exp \left(54,435096 i_{10}{ }^{0,0190103}\right) .
$$

Next check the adequacy of the models (4) and (9). Known formulas allowing to calculate the number of primes faster. In this way, it was calculated that up to $10^{23}$ is 1925320391606803968923 primes.

Then we get to the data [1] the new value $x / \pi(x)=51,9394$ (Table 5).

Model (9), obtained from the physical constants in Table 4, was even more precise on the relative error, and it gives more accurate predictions of the relative power of the set of prime numbers. 
The error for the array $i_{10}=23$ is equal to only $0,08 \%$.

By the remnants of (9) is obtained (Figure 8) the equations of the perturbation.

Table 5. The adequacy of the law of exponential growth

\begin{tabular}{|c|c|c|c|c|c|c|c|}
\hline \multirow{2}{*}{$\begin{array}{c}\text { Discharge } \\
i_{10}\end{array}$} & \multirow{2}{*}{$\frac{x}{\pi(x)}$} & \multicolumn{3}{|c|}{ Model (4) } & \multicolumn{3}{|c|}{ Model (9) } \\
\hline & & $x / \pi(x)$ & $\varepsilon$ & $\Delta, \%$ & $x / \pi(x)_{f}$ & $\varepsilon$ & $\Delta, \%$ \\
\hline 1 & 1.6667 & 1.8420 & -0.1753 & -10.52 & 1.8330 & -0.1663 & -9.98 \\
\hline 2 & 3.7037 & 3.8481 & -0.1444 & -3.90 & 3.7735 & -0.0698 & -1.88 \\
\hline 3 & 5.8824 & 5.9481 & -0.0657 & -1.12 & 5.7822 & 0.1002 & 1.70 \\
\hline 4 & 8.1235 & 8.1181 & 0.0054 & 0.07 & 7.8429 & 0.2806 & 3.45 \\
\hline 5 & 10.4232 & 10.3452 & 0.0780 & 0.75 & 9.9462 & 0.4770 & 4.58 \\
\hline 6 & 12.7389 & 12.6211 & 0.1178 & 0.92 & 12.0861 & 0.6528 & 5.12 \\
\hline 7 & 15.0471 & 14.9398 & 0.1073 & 0.71 & 14.2584 & 0.7887 & 5.24 \\
\hline 8 & 17.3567 & 17.2969 & 0.0598 & 0.34 & 16.4598 & 0.8969 & 5.17 \\
\hline 9 & 19.6666 & 19.6890 & -0.0224 & -0.11 & 18.6876 & 0.9790 & 4.98 \\
\hline 10 & 21.9755 & 22.1132 & -0.1377 & -0.63 & 20.9399 & 1.0356 & 4.71 \\
\hline 23 & 51.9394 & 55.8321 & -3.8927 & -7.49 & 51.8993 & 0.0401 & 0.08 \\
\hline
\end{tabular}

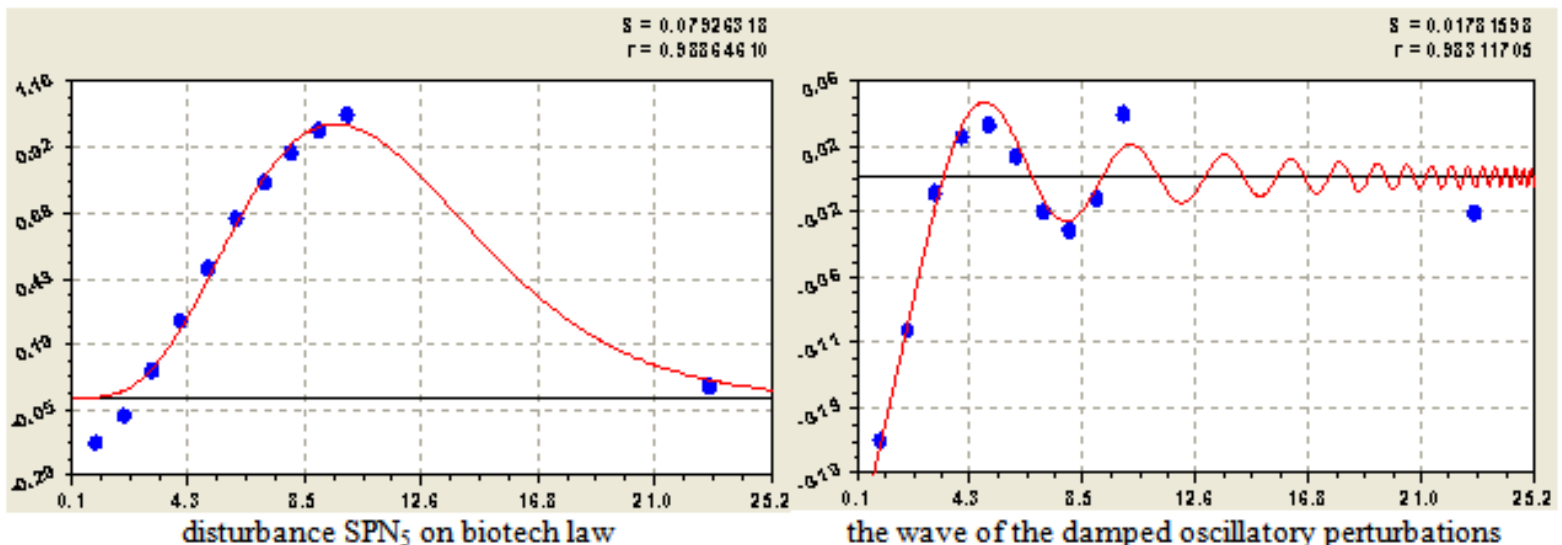

Figure 8. Diagrams of the perturbation capacity of prime numbers depending on the order of the decimal system

Biotechnical law as a supplement to (9) shows that after the discharge $i_{10}=23$ in the relative power is going on a decline. Damped oscillation shows that with increasing power of primes wave $x / \pi(x)$ tends to zero. When $i_{10}>>23$ the perturbation is almost excluded.

\section{Conclusions}

Power total number of primes from the discharge of the decimal system is identified by the law of exponential growth to the fundamental physical constants. With the growth of the power of the prime numbers increases the adequacy of equation (8) with the physical constants, which can lead in the future to the general equation four interactions.

\section{References}

[1] Don Zagier. The first 50 million prime numbers. URL: http://www.ega-math.narod.ru/Liv/Zagier.htm.

[2] Number. URL: http://ru.wikipedia.org/wiki/\%D0\%A7\%D0\%B8\%D1\%81\%D0\% BB\%D0\%BE.

[3] Mazurkin P.M. Biotechnical principle and sustainable laws of distribution // Successes of modern natural sciences. 2009. № 9, 93-97.

[4] Mazurkin PM The statistical model of the periodic system of chemical elements D.I. Mendeleev. Yoshkar-Ola: MarSTU, 2006. 152.

[5] Fundamental physical constants. URL; http://www.akin.ru/spravka/s_fund.htm. 